\title{
Bias-dependent amino-acid-induced conductance changes in short semi-metallic carbon nanotubes
}

\author{
G.B. Abadir, K. Walus and D.L. Pulfrey \\ Department of Electrical and Computer Engineering \\ The University of British Columbia, Vancouver, BC, V6T1Z4, Canada \\ E-mail: gabadir@ieee.org
}

\begin{abstract}
.
We study the interaction between short semi-metallic carbon nanotubes and different amino acids using molecular dynamics and ab initio (density functional theory/non-equilibrium Green's function) simulations. We identify two different mechanisms of nanotube-conductance-change upon adsorption of amino acids: one due to the change of the coordinates of the nanotube arising from van der Waals interaction forces with the adsorbed amino acid; and one due to electrostatic interactions, which appear only in the case of charged amino acids. We also find that the transport mechanism and the changes in the conductance of the tube upon amino-acid adsorption are bias-dependent.
\end{abstract}

PACS number: 72.80.RJ

Submitted to: Nanotechnology 


\section{Introduction}

Carbon nanotubes (CNTs) have been previously shown to exhibit large changes in conductance in response to biomolecule adsorption $[1,2,3,4,5]$. The experimental work involving biosensing using such changes has been performed using semiconducting CNTs in a three-terminal field-effect-transistor (FET) configuration. The gate of the FET can be either a metallic back-gate, or a liquid gate that includes the analyte. Back-gated devices are rather complicated to fabricate, and liquid-gated devices require precise control of the gate potential. Also, the exact mechanism of interaction is not yet quite clear. Several suggestions have been made: electrostatic gating as the detected molecules change the potential of the gate in the case of liquid gating [6], electron donation by the detected species [1], reduction in carrier mobility [7], liquid-gate-capacitance changes [8], and changes in the Schottky barrier height when the adsorption occurs near the electrode-CNT interface [9, 10].

In this paper, we study the effect of amino-acid adsorption on the conductance of single-walled carbon nanotubes in a simple two-probe configuration. We choose to study semi-metallic tubes (tubes with very small bandgaps) as they have a higher possibility of conductance modulation in such a two-probe configuration than either metallic tubes with zero gaps, where no change in the current is expected (and that is confirmed by calculations for armchair tubes that are not presented in this paper), or semiconducting tubes which need a much higher bias to cause any appreciable current at all. We choose amino acids as they are the building blocks of proteins, and because understanding their interaction with CNTs will help to understand how full proteins (which are too complex to be presently simulated by the state-of-the-art $a b$ initio techniques used here) interact with CNTs.

We use molecular dynamics (MD) simulations to get the relative coordinates of the CNT and the adsorbed biomolecule. The results are then used in ab initio density functional theory/non-equilibrium Green's function (DFT/NEGF) simulations [11] within the local density approximation (LDA) [12]. Our study considers the semimetallic $(9,0),(12,0),(15,0)$, and $(18,0)$ tubes. For the amino acids, our study covers a range of amino acids of different charge types. We used dimers of the neutral aromatic amino acids tyrosine (TYR), tryptophan (TRP), and phenylalanine (PHE), as well as the positively charged amino acid arginine (ARG), the negatively charged amino acids glutamate (GLU) and aspartate (ASP), and the two neutral non-aromatic amino acids asparagine (ASN), and isoleucine (ILE).

\section{Simulated Structures}

The simulation flow was as follows: MD simulations of five periods $(\sim 2.2 \mathrm{~nm})$ of each of the nanotubes in water, with and without the amino-acid dimers, were first performed using the package GROMACS [13]. The AMBER99 port [14] and the TIP3P model [15] were used for the amino acids and the water, respectively, and simulations were run for 
$2 \mathrm{~ns}$ to get the relative coordinates of the atoms of the nanotubes and the dimers. The pressure was held at 1 atm using Parrinello-Rahman coupling [16], and the temperature was maintained at $300 \mathrm{~K}$ using Berendsen coupling [17]. A Morse potential was used to describe the bonds of the CNT and a cosine potential was used to describe the angles. These parameters were previously used in MD simulations of carbon nanotubes in the presence of biomolecules [18], and were found to reproduce experimental results successfully. Counterions were included with the charged amino acids to neutralize the net charge (sodium ions with glutamate and aspartate, and chlorine ions with arginine). The coordinates were then fed to the DFT/NEGF simulator Atomistix (ATK) [19]. In ATK, two-probe simulations were performed where the coordinates of the central region were those calculated from the MD simulations as explained above. It is important to note that the coordinates cannot be simply obtained through DFT optimization because DFT does not account for van der Waals forces [20, 21], which play an important role in the interaction between nanotubes and amino acids [20, 22]. The central region was sandwiched between two semi-infinite CNT electrodes of the same chirality as the nanotube in the central region.

The basis set used in the $a b$ initio simulations can significantly affect the results, even qualitatively, as we have previously demonstrated [23, 24]. Therefore, we first made a test with the $(9,0)$ tube, where we repeated the bare-tube simulations and the simulations with arginine using the double-zeta (DZ), double-zeta polarized (DZP), and single-zeta-polarized (SZP) basis sets. We found that the DZP basis set predicts the largest relative current change due to the adsorption of the ARG dimer, and that the difference between the relative current changes predicted by the DZ and the SZP basis sets is not significant in the range of bias used. In addition, the DZ basis set predicts the total charge on the tube and the amino acid in excellent agreement with that predicted with the DZP basis set, whereas the predictions obtained using the SZP basis set are significantly different, as was previously demonstrated [24]. An accurate prediction of the total charge is particularly important for this study since we are inspecting charged species as well as neutral ones. Hence, and since larger-radius tubes could not be simulated using the DZP basis set due to memory limitations, we used the DZ basis set throughout this paper even though this may underestimate the effect of the amino acid.

In addition, ATK uses periodic boundary conditions in the directions transverse to the transport direction, meaning that the simulation box is repeated indefininitely in the transverse directions. To assure that no significant interaction occurs between the actual simulation box and its repeated images as was previously pointed out [25], the transverse dimensions of the simulation box were increased for each simulation until no significant change $(\sim 1 \%)$ in the calculated current was observed.

Finally, a screening region is introduced in ATK to isolate the central region from the electrodes to assure charge neutrality in the electrodes. This is because the electrodes are assumed to be extended infinitely in the transport direction, and the repeated images of the electrode are assumed to have the same charge distribution as the electrode cell in the actual simulation box. The screening-region length (SRL) was chosen as one period 
(four layers of atoms) of the tube between the central region and each of the electrodes. Then, the SRL was doubled for the $(9,0)$ bare tube and the $(9,0)$ tube with the adsorbed arginine; and no change in the electron density in the elctrodes was observed. In fact, the electron density in the electrodes in both cases was almost identical to an infinitelylong bare tube of the same chirality. Also, there was no significant change in the current/voltage characteristics as will be shown in the results below. Using a longer SRL for the larger tubes (especially the $(15,0)$ and the $(18,0)$ tubes) was not possible even with the DZ basis set due to memory limitations. Therefore all simulations presented here were performed using a four-layer SRL.

\section{Results and discussion}

From the MD simulations, each of the dimers was adsorbed on all the different nanotubes, except for those of glutamate and aspartate, which failed to be adsorbed on any of the tubes, and therefore were not considered in the DFT/NEGF simulations. The other amino acids settled at a distance of $0.25 \mathrm{~nm}$ to $0.29 \mathrm{~nm}$ from the surface of the tubes. The counterions settled at a distance more than $1.5 \mathrm{~nm}$ away from the surface of all the tubes and therefore were neglected in the DFT/NEGF simulations. The current (I)-voltage (V) characteristics of the $(9,0)$ tube are shown in Figure 1 for the bare tube and for the same tube with an adsorbed dimer of each of the aromatic amino acids used. It is clear that in all the cases the current increases with bias, then virtually saturates until $V$ exceeds $E_{G} / q$ where $q$ is the electronic charge and $E_{G}$ is the bandgap of the tube. When $V$ exceeds $E_{G} / q$, the current starts to increase again with the applied bias. This indicates the onset of a new carrier transport mechanism (namely, band-toband tunneling), as will be explained below in the transport mechanisms discussion. It is also clear that these adsorbed aromatic amino acids do not have any significant effect on the conductance of the tube. It is interesting to note that our results for the interaction between aromatic amino acids and semiconducting tubes (not shown here) demonstrate a clear change in the conductance of semiconducting tubes upon amino-acid adsorption. A similar change had been observed experimentally with other aromatic molecules [26], and had been attributed to charge transfer between the tube and the aromatic molecules. Although our calculations show that a similar charge transfer occurs between the aromatic amino acids and the semi-metallic tubes, it does not have the same

effect as it has with the semiconducting ones. A further study is needed to explain the reason behind this difference in response between the two types of tube.

Figure 2 shows the I-V charcteristics of the $(9,0)$ tube, both bare and with an adsorbed dimer of each of the neutral non-aromatic amino acids isoleucine and asparagine, as well as with an adsorbed dimer of arginine. Again, with the non-aromatic neutral amino acids, and the positively charged amino acid arginine, we observe the same trend of the current with increasing bias as mentioned above. We also notice that the effect of the adsorbed amino acid on the current is bias-dependent. ASN and ILE adsorption results in a small reduction in current when $V<E_{G} / q$, while it has no effect 
for higher bias levels. ARG induces a larger decrease in the current for $V<E_{G} / q$, but also causes a clear increase in the current when $V$ exceeds $E_{G} / q$. The I-V characteristics of the $(18,0)$ tube were also calculated in the presence of neutral amino acids (results not shown here), and again, no significant change in the current was observed. As for ARG, Figure 3 shows the I-V characteristics of the various tubes with and without an adsorbed dimer of this positively charged amino acid. It is evident that for all the tubes, the conductance $(G=I / V)$ decreases upon the adsorption of the arginine dimer compared to the corresponding bare-tube current for $V<E_{G} / q$. When $V$ exceeds $E_{G} / q$, the observed suppression of the current upon ARG adsorption starts to decrease, and eventually the current with the adsorbed amino acid becomes larger than the bare-tube current, indicating an increase in $G$. We wish to emphasize here that increasing the SRL discussed above in section 2 had no effect on the current of the bare tube in both bias

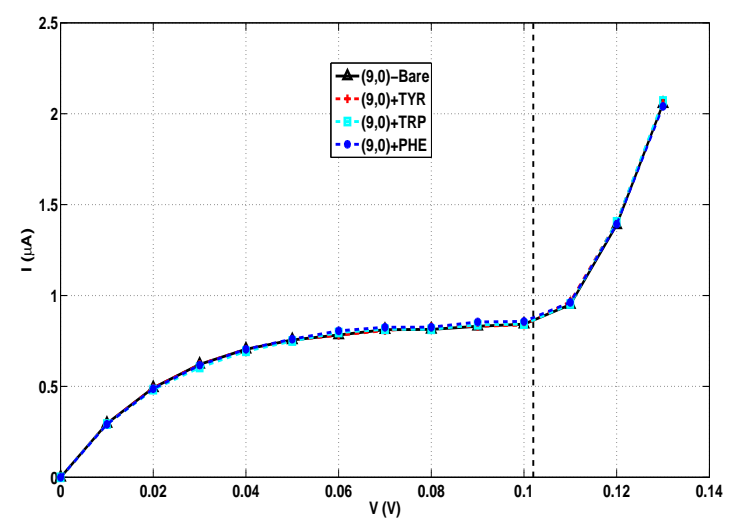

Figure 1. I-V characterstics for the bare $(9,0)$ tube and the same tube with an adsorbed dimer of each of the aromatic amino acids TYR, TRP, and PHE. The vertical line indicates the bias value at which $V=E_{G} / q$.

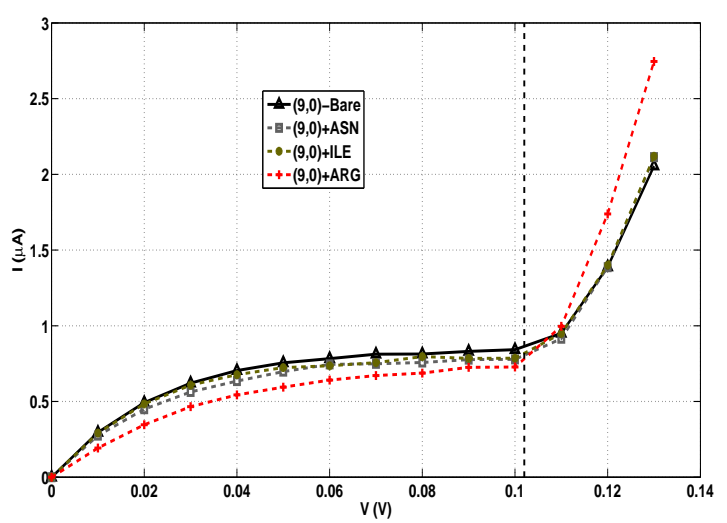

Figure 2. I-V characterstics for the bare $(9,0)$ tube and the same tube with an adsorbed dimer of each of the neutral non-aromatic amino acids ASN and ILE, and the positively-charged amino acid ARG. The vertical line indicates the bias value at which $V=E_{G} / q$. 


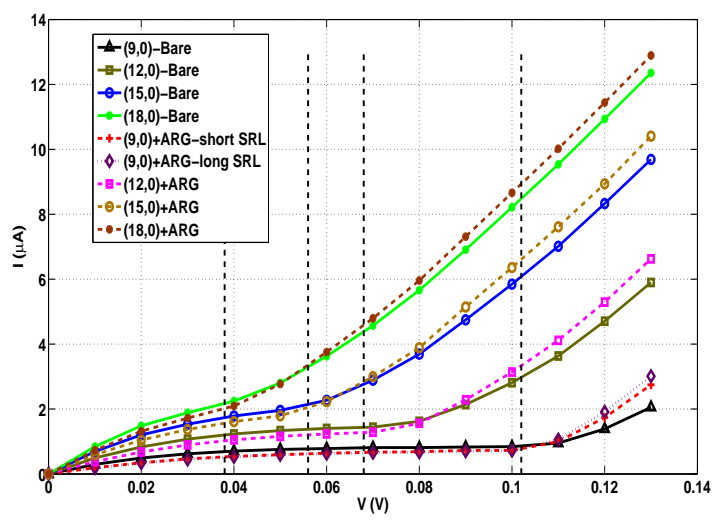

Figure 3. I-V characterstics for the bare tubes and for the tubes with an adsorbed dimer of arginine. From left to right, the vertical lines denote the bias value at which $V=E_{G} / q$ for the $(18,0),(15,0),(12,0)$, and the $(9,0)$ tubes, respectively.

ranges. For the tube with the adsorbed arginine, increasing the SRL had no signifcant effect in the first bias range $\left(V<E_{G} / q\right)$, while in the second bias range $\left(V>E_{G} / q\right)$, there was a small increase in the current when the SRL was increased compared to the shorter-SRL case, as can be seen in the bottom set of curves in Figure 3. Taking into account that in this range the current for the short-SRL case is already greater than that of the bare tube, we can see that using the shorter SRL in the simulations gives a slightly lower estimation of the change in the current upon arginine adsorption. We conclude that using the short SRL does not compromise our conclusions, while permitting the study of the larger tubes as explained in section 2 .

The effect of ARG adsorption on the conductance of the various tubes is summarized in Figure 4. It is clear that the change in the current relative to the bare-tube case decreases with the increasing radius of the tube. This may be explained by the fact that the background current (i.e., the current of the bare tube) is larger (at the same bias) for larger-radius tubes due to their smaller bandgap and, therefore, higher intrinsic carrier density. Therefore, among the semi-metallic tubes, which are expected to have a higher possibility for conductance modulation than their semiconducting and metallic counterparts, those with smaller radii are expected to exhibit the larger responses to amino-acid adsorption.

\section{Transport mechanisms}

Here, we introduce Figure 5 to offer an explanation for the features of the I-V characteristics. When the bias (in volts) is numerically lower than the bandgap (in electron volts), electrons can move from the conduction band in the left electrode to free states in the conduction band in the right electrode creating a left-to-right current $\left(I_{L R}\right)$. Similarly, electrons in the conduction band of the right electrode can move to free states in the left electrode creating a right-to-left current $\left(I_{R L}\right) . I_{R L}$, however, will 
be smaller than $I_{L R}$ due to the energy barrier $(q V)$ that the electrons moving from the right electrode to the left electrode encounter. The net flow of electrons creates what we will refer to as the intraband current $\left(I_{I B}\right)$. As the bias increases, the energy barrier increases and therefore $I_{R L}$ decreases until it vanishes, leading to the eventual saturation of the net current $I_{I B}$. As long as the bias is numerically less than the bandgap, electrons in the valence band of the left electrode cannot tunnel through to the right electrode as there are no available states at the corresponding energies on the right electrode. When the bias numerically exceeds the bandgap, however, free states in the conduction band of the right electrode become available for electrons in the valence band of the left electrode to tunnel into and contribute to a band-to-band-tunneling current $\left(I_{B T B}\right)$. This tunneling current is the reason behind the sudden increase in the current when $V>E_{G} / q$. This is further confirmed in Figure 6, which depicts the transmission coefficient as a function of energy $(T(E))$ of the $(9,0)$ tube at different bias points in the two bias regions described above. When the bias is $0.01 \mathrm{~V}$ (much less than $E_{G} / q$ ), there is no transmission probability for energies within the bandgap, which results in a zero transmission coefficient. When the bias exceeds $E_{G} / q$, on the other hand, we can see a sizeable transmission coefficient within the bandgap, which is associated with $I_{B T B}$ as explained above. The same phenomenon is also observed for the $(12,0),(15,0)$, and the $(18,0)$ tubes, (the corresponding transmission coefficients are not shown here). This tunneling current may also explain the unexpectedly high current levels that were observed previously in similar simulations [27]. We remark here that although a first glance at Figure 6 may appear to indicate that the bandgap widens with increasing the bias, this is not actually the case. $T(E)$ represents the sum of the probabilities of transmission between the two electrodes for different eigenchannels. Since in the absence of tunneling no transmission can occur in the energy window between the quasi-Fermi levels of the electrodes, the transmission coefficient in this

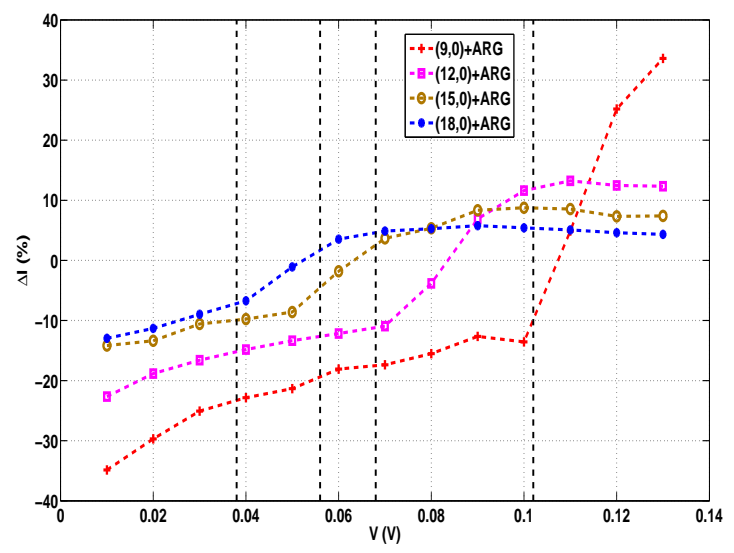

Figure 4. Relative change in the current compared to the current of the bare tubes upon ARG adsorption. From left to right, the vertical lines denote the bias value at which $V=E_{G} / q$ for the $(18,0),(15,0),(12,0)$, and the $(9,0)$ tubes, respectively. 


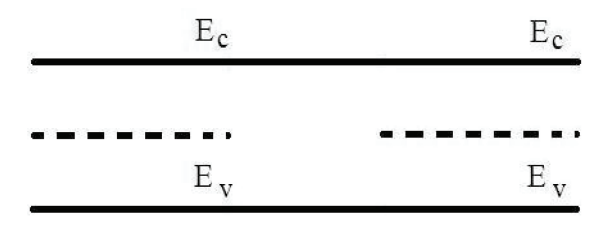

(a)

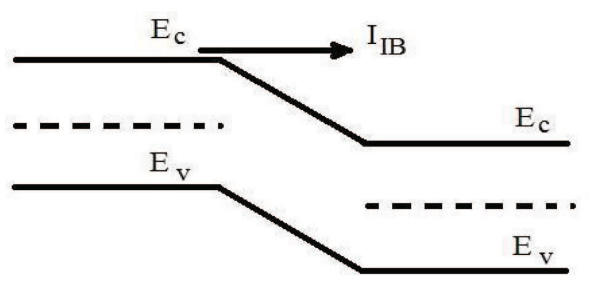

(b)

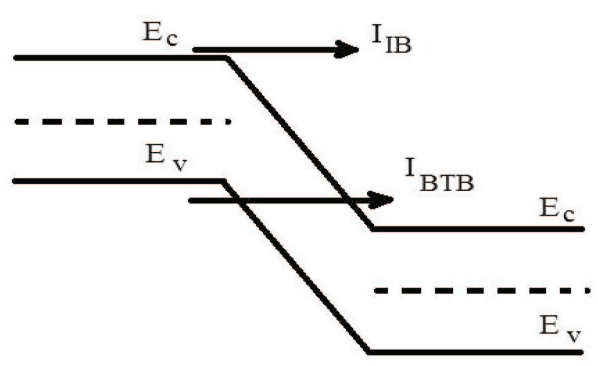

(c)

Figure 5. Schematic band diagram for the bare tube for (a) $V=0$, (b) $0<V<E_{G} / q$, and (c) $V>E_{G} / q$. The dashed lines depict the Fermi level or quasi-Fermi level in each of the contacts.

window is essentially zero except for the energy values at which band-to-band tunneling is possible. It is worth noting that, in practice, fabrication of intrinsic CNTs with the Fermi level in the middle of the gap as shown in Figure 5 can be achieved through the dry contact transfer method [28]. For cases where the Fermi level lies inside the conduction band, the intraband current of the bare tube is expected to increase because of the larger number of carriers available in the conduction band. This may lead to a decrease in the relative change caused by the analyte before the onset of the tunneling. If the Fermi level lies inside the valence band, the opposite is expected to occur: the bare-tube intraband current is expected to decrease, and the effect of the analyte, in relative terms, should be greater. The tunneling current should not be affected in either case.

\section{Mechanisms of conductance change}

We discuss here the changes in the current upon ARG adsorption. When the bias is numerically less than the bandgap of the tube, the proximity of the charged amino acid to the nanotube disturbs the electrostatic potential in the central region. The irregularity 


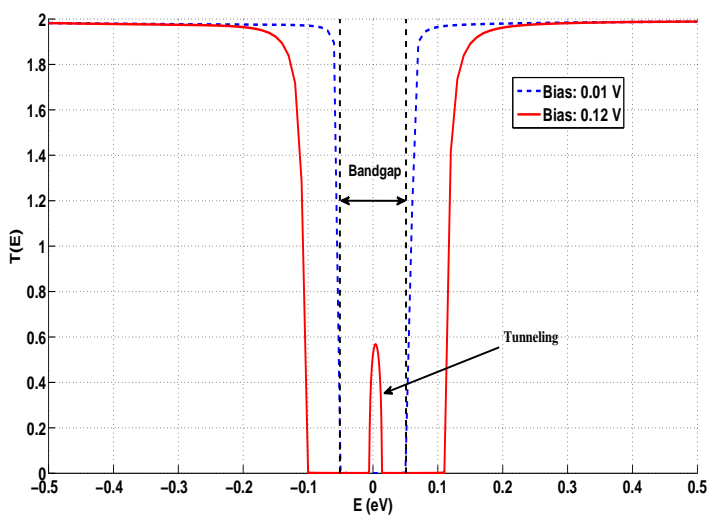

Figure 6. Transmission coefficient of the bare $(9,0)$ at two bias points: $0.01 \mathrm{~V}$ and $0.12 \mathrm{~V}$.

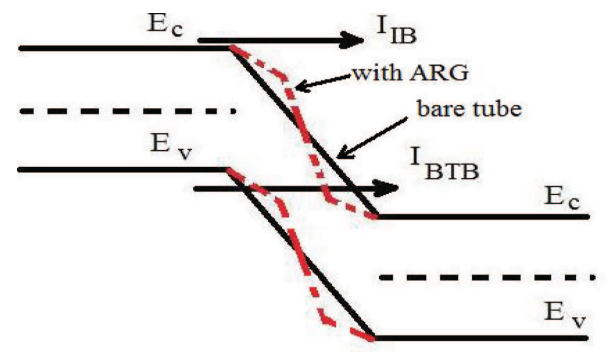

Figure 7. Schematic band diagram illustrating the effect of the adsorption of ARG on the electrostatic potential energy in the CNT for $V>E_{G} / q$.

of the potential causes quantum mechanical reflections that reduce the transmission probability between the two electrodes and therefore $I_{I B}$ decreases.

For the range where $V>E_{G} / q$, the observed increase in the current can be understood from Figure 7. The charged amino acid increases the field locally, thus decreasing the effective width through which electrons should tunnel, as schematically shown in Figure 7, resulting in an increase in the tunneling probability and hence in $I_{B T B}$.

To justify the explanation above, we calculated the total current $\left(I_{t o t}\right)$ and $I_{I B}$ for the various tubes with and without $\mathrm{ARG}$, as well as the $I_{B T B}$ component of the total current. The results are shown in Table 1 for the $(9,0)$ and $(12,0)$ tubes. Table 1 shows that the presence of ARG suppresses $I_{I B}$ while enhancing $I_{B T B}$. A similar behaviour was also observed for the $(15,0)$ and $(18,0)$ tubes.

To further corroborate the suggested explanation, we calculated the electrostatic potential $V_{E S}$ in the central region for the simulation with the bare $(9,0)$ tube (CNTbare) and the same tube with the adsorbed arginine (CNT-ARG). A third simulation (CNT-ARG-bare) was also performed with the coordinates of the CNT atoms being identical to those in CNT-ARG, but with the atoms of the amino acid being omitted in the DFT/NEGF simulation. In other words, the amino acid atoms were included in the 
MD simulations but not in the subsequent DFT/NEGF simulations for CNT-ARG-bare. The purpose is to examine whether the suggested irregularities in the potential are a result of the tube deformation due to van der Waals interaction forces with the amino acid, or whether electrostatic interactions are the actual reason for those irregularities. Figure 8(b) shows the difference in $V_{E S}\left(\Delta V_{E S}\right)$ between CNT-ARG and CNT-bare as well as between CNT-ARG-bare and CNT-bare along the line directly under the adsorbed dimer as illustrated in Figure 8(a). We can see that for both CNT-ARG and CNT-ARG-bare, there is a change in the electrostatic potential. The change in the case of CNT-ARG, however, is significantly greater near the two extremities of the central region, and decreases near the middle of the central region to become almost identical to that associated with CNT-ARG-bare. This can be explained as follows: the charge on the dimer resides on the guanidino groups on each of the arginine residues [29] as confirmed by Mulliken population analysis at the end of the DFT/NEGF simulation. The guanidino groups stretch over the tube near the two extremities of the central regions as shown in Figure 8(a). Therefore, the effect of the charge is more pronounced near the guanidino groups and decays as we move towards the middle of the central region. A similar calculation was conducted along a line at the bottom of the tube (on the opposite side of the adsorption site of the dimer), and $\Delta V_{E S}$ was identical for both CNT-ARG and CNT-ARG-bare along that latter line. The results show that

Table 1. Different components of the current for the $(9,0)$ and $(12,0)$ tubes with and without the adsorbed dimer of arginine, and at the two bias points $0.01 \mathrm{~V}$ and $0.12 \mathrm{~V}$. Currents less than $1 \mathrm{nA}$ were considered to be exactly zero.

\begin{tabular}{|c|c|c|c|c|}
\hline structure & $I_{I B}(n A)$ & $I_{B T B}(n A)$ & $I_{t o t}(n A)$ & $\frac{I_{B T B}}{I_{\text {tot }}}(\%)$ \\
\hline $\begin{array}{l}(9,0) \text {-bare: } \\
0.01 \mathrm{~V}\end{array}$ & 295.77 & 0.00 & 295.77 & 0.00 \\
\hline $\begin{array}{l}(9,0)-A R G: \\
0.01 \mathrm{~V}\end{array}$ & 202.30 & 0.00 & 202.30 & 0.00 \\
\hline $\begin{array}{l}(9,0) \text {-bare: } \\
0.12 \mathrm{~V}\end{array}$ & 922.80 & 465.90 & 1388.70 & 33.55 \\
\hline $\begin{array}{l}(9,0)-A R G: \\
0.12 \mathrm{~V}\end{array}$ & 823.25 & 826.25 & 1649.50 & 50.09 \\
\hline $\begin{array}{l}(12,0) \text {-bare: } \\
0.01 \mathrm{~V}\end{array}$ & 492.48 & 0.00 & 492.48 & 0.00 \\
\hline $\begin{array}{l}(12,0)-A R G: \\
0.01 \mathrm{~V}\end{array}$ & 380.90 & 0.00 & 380.90 & 0.00 \\
\hline $\begin{array}{l}(12,0) \text {-bare: } \\
0.12 \mathrm{~V}\end{array}$ & 1540.90 & 3165.60 & 4706.50 & 67.26 \\
\hline $\begin{array}{l}(12,0)-A R G: \\
0.12 \mathrm{~V}\end{array}$ & 1428.30 & 3865.20 & 5293.50 & 73.02 \\
\hline
\end{tabular}




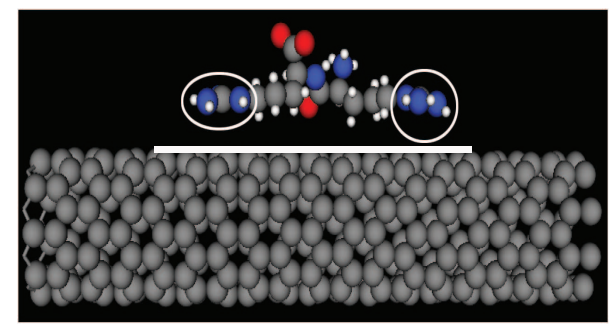

(a)

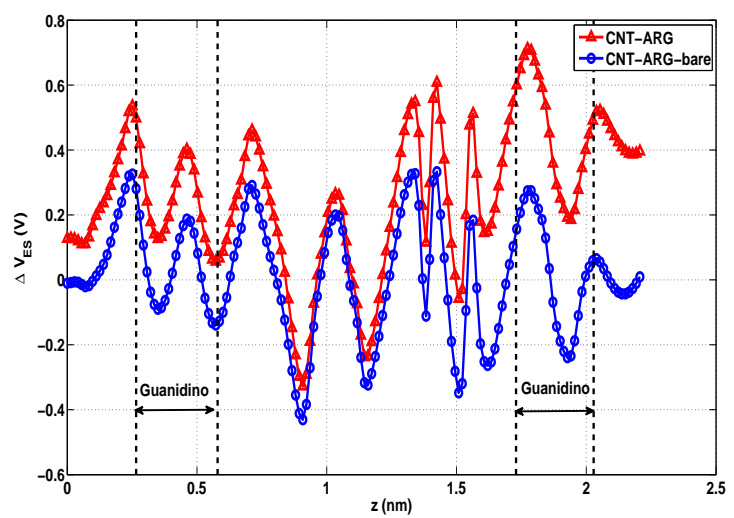

(b)

Figure 8. Difference in the electrostatic potential in the central region between CNT-ARG and CNT-ARG-bare on one hand, and CNT-bare on the other hand. The difference is shown in (b) along the line shown in (a). The ellipses in (a) denote the guanidino groups.

both the change in the coordinates of the tube due to van der Waals forces, and the electrostatic interactions due to the charge on the amino acids contribute to $\Delta V_{E S}$, and result in local disturbances in the electrostatic potential. The similarity in the general shape of the two curves in Figure 8(b) indicates that this general shape is caused by the change in the coordinates, not by the electrostatic interactions. The electrostatic interactions, however, induce a significant local increase in $\Delta V_{E S}$, leading to further local disturbances in the electrostatic potential. The local potential disturbances result in quantum mechanical reflections, thus decreasing the transmission probability between the two electrodes, and consequently decreasing the conductance of the tube.

For the increase in the tunneling, we show the LDOS at an energy of $0.0 \mathrm{eV}$ (same reference energy in Figure 6), and a bias of $0.12 \mathrm{~V}$, on a cut-plane perpendciular to the transport direction as shown in Figure 9. We can see a clear increase in the LDOS upon ARG adsorption in comparison with the bare tube. An increase in the LDOS was observed all along the tube, but the LDOS closer to the amino acid (upper part of Figure 9(c)) was higher than away from the adsorbed amino acid. We note that the maximum value of the LDOS is $0.03006 \AA^{-3} \mathrm{eV}^{-1}$ for the bare tube compared to $0.1236 \AA^{-3} \mathrm{eV}^{-1}$ with ARG. The increase in the LDOS is in agreement with the 
lowering of the potential energy and the consequent increase in the tunneling probability suggested above.

For significantly longer tubes, the tunneling current will naturally cease to contribute to the total current, and only the intraband current will exist. For such tubes, and as far as the transport is still ballistic ( $\sim$ tens of nanometers [30, 31]), the quantum mechanical reflections are expected to cause a noticeable change in the current just as in the short tubes studied here. For much longer tubes $(\sim \mu \mathrm{m})$, where other scattering mechanisms (e.g., phonon scattering [30,31]) are present, a separate study is needed to assess the effect of quantum mechanical reflections on the I-V characteristics of such tubes.

We also note that at such a short length of the device, high electric fields may arise and there may be a possibility of breakdown, specially in the band-to-bandtunneling operation region described above. We demonstrate here that this possibility is not of serious concern for our structures. The breakdown of carbon nanotubes has been attributed to heating associated with phonon-electron scattering [32, 33]. Due to ballistic transport in the short tubes presented in this work, and to the fact that they are shorter than the phonon mean-free path [30], such a scattering is impossible. Also, a value of the field close to that reported here was reported in [30], and no breakdown was observed. Therefore, the possibility of breakdown is excluded.

We performed two further simulations similar to CNT-ARG-bare described above, but with ASN and ILE instead of ARG, as these amino acids resulted in a visible (though small) effect on the I-V characteristics of the $(9,0)$ tube. We will refer to these simulations as CNT-ASN-bare and CNT-ILE-bare. The purpose is again to examine if the change in the current is due to changes in the coordinates of the CNT atoms resulting from van der Waals forces between the tube and the amino acid, or if other effects (e.g., Coulombic interactions) are more important in changing the current. The results are shown in Figure 10 together with the results for CNT-bare, CNT-ARG, and CNT-ARG-bare for comparison. The simulations that include the atoms of ASN and ILE in both MD and DFT/NEGF simulations will be referred to as CNT-ASN and CNT-ILE, respectively. The results for CNT-ARG-bare compared to CNT-bare show that the current still changes, although the changes observed with CNT-ARG are clearly much more pronounced. This suggests that the potential disturbances due to the electrostatic interactions discussed above are the main reason behind the changes in the current, and that those disturbances caused by the change in the coordinates of the tube atoms play a minor role. For the neutral amino acids ASN and ILE, the results of CNT-ASN-bare and CNT-ILE-bare are very close to CNT-ASN and CNT-ILE. This shows that the van der Waals interaction forces between the amino acids and the tube, and the resulting coordinate changes of the tube atoms, are the reason for the observed small conductance changes in this case. The aromatic amino acids had virtually no effect at all on the conductance of the tube. This is because of two reasons: the first one is that they are neutral and therefore have no Coulombic interactions, and the second one is that aromatic amino acids adsorb on the nanotubes through $\pi$-stacking, i.e., the 


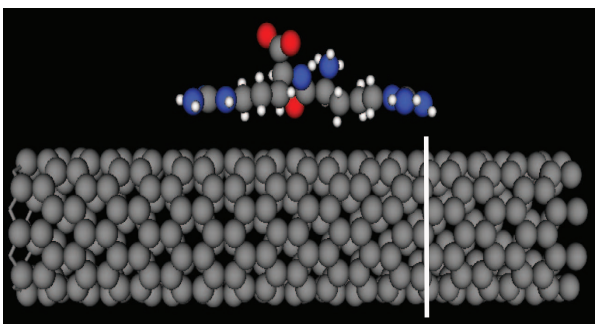

(a)

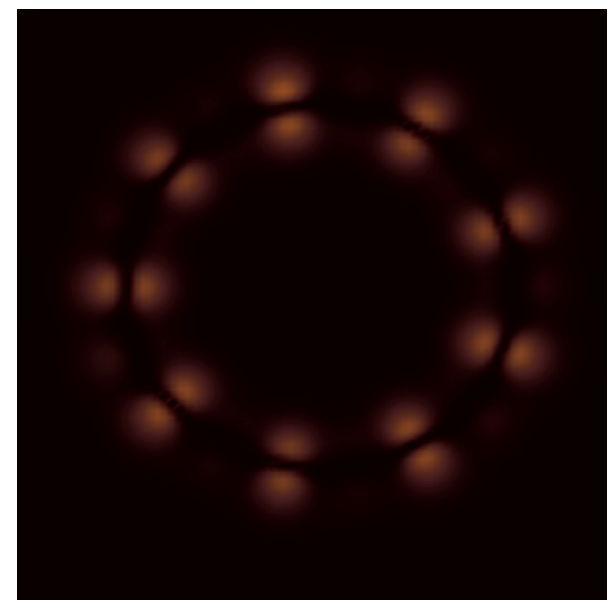

(b)

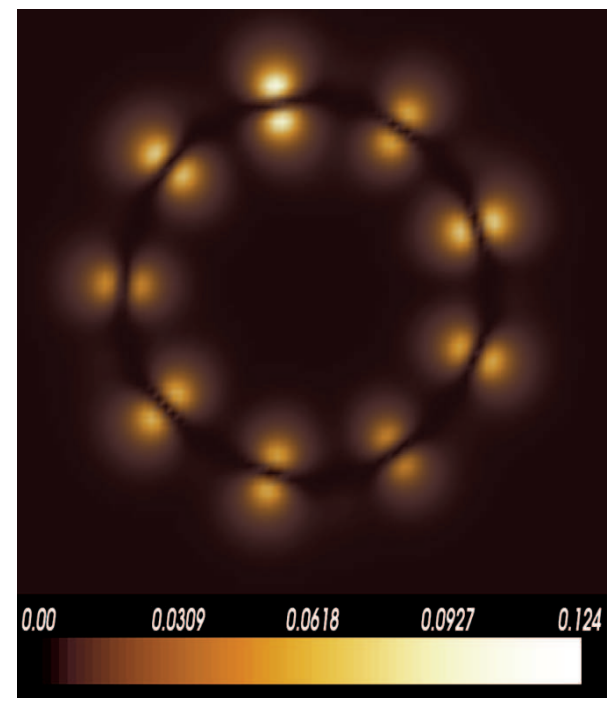

(c)

Figure 9. LDOS at an energy of $0.0 \mathrm{eV}$, and a bias of $0.12 \mathrm{~V}$, in units of $\AA^{-3} \mathrm{eV}^{-1}$ on a cut-plane perpendicular to the transport direction at the line shown in (a) for (b) bare tube, (c) tube with adsorbed arginine. 


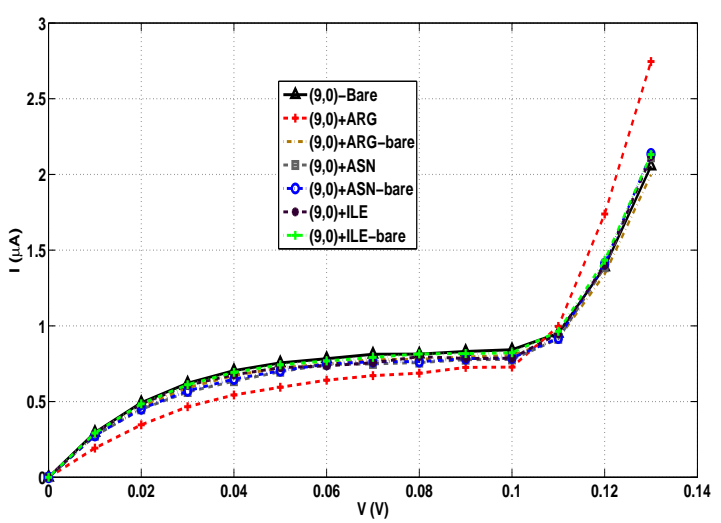

Figure 10. I-V characteristics for CNT-ARG-bare, CNT-ASN-bare, CNT-ILE-bare. The results for CNT-ARG, CNT-ASN, CNT-ILE, and CNT-bare are also included for comparison.

aromatic ring stacks over the rings already present in the tube. Therefore, the changes in the coordinates of the tubes are minimal and do not cause any significant effect on the conductance of the tube.

\section{Conclusion}

In this work we demonstrated that short semi-metallic carbon nanotubes show a clear change in conductance in response to adsorption of the positively charged amino acid arginine, while neutral aromatic and non-aromatic amino acids have little effect on the conductance of such tubes. We also showed that these tubes exhibit two different transport mechanisms depending on the applied bias: tunneling current becomes a significant component of the total current when the applied bias (in volts) numerically exceeds the bandgap of the tubes (in electron volts). The effect of arginine on the conductance of the tubes is also dependent on the applied bias. When the bias is numerically lower than the bandgap of the tube, an amino-acid-induced disturbance of the electrostatic potential causes quantum mechanical reflections that decrease the conductance of the tube, while at higher bias levels, the tunneling probability increases due to the presence of arginine. The results demonstrate that non-functionalized short semi-metallic tubes show a high selectivity towards charged species, and that they may be useful as biosensors in a simple two-terminal configuration.

\section{Acknowledgments}

This work was supported by the Natural Sciences and Engineering Research Council of Canada (NSERC) and the Canadian Foundation for Innovation (CFI). We also thank Dr. Anders Blom of Quantumwise, the new supplier of the DFT/NEGF software used in this work, for his continuous support. 


\section{References}

[1] Gruner G 2006 Anal. Bioanal. Chem. 384 322-335.

[2] Allen B, Kichambare P and Star A 2006 Adv. Mater. 19 1439-51.

[3] Balasubramanian K and Burghard M 2006 Anal. Bioanal. Chem. 385 452-468.

[4] Wang J 2005 Electroanal. 17 7-14.

[5] Tang X, Bansaruntip S, Nakayama N, Yenilmez E, Chang Y and Wang Q 2006 Nano Lett. 6 1632-36.

[6] Heller I, Janssens A M, Mannik J, Minot E D, Lemay S G and Dekker C 2008 Nano Lett. 8 591-595.

[7] Maroto A, Balasubramanian K, Burghard M and Kern K 2007 ChemPhysChem 8 220-223.

[8] Besteman K, Lee J, Wiertz F G M, Heering H A and Dekker C 2003 Nano Lett. 3 727-730.

[9] Chen R J, Choi H C, Bangsaruntip S, Yenilmez E, Tang X, Wang Q, Chang Y L and Dai H 2004 J. Am. Chem. Soc. 126 1563-68.

[10] Dong X C, Fu D L, Xu Y P, Wei J Q, Shi Y M, Chen P and Li L J 2008 J. Phys. Chem. C 112 9891-95.

[11] Stokbro K, Taylor J, Brandbyge M and Guo H 2005 Ab-initio Non-Equilibrium Green's Function Formalism for Calculating Electron Transport in Molecular Devices Introducing Molecular Electronics (lecture Notes in Physics) ed G Cuniberti, G Fagas and K Richter (Springer: Berlin/Heidelberg) pp 117-152.

[12] Perdew J P and Zunger A 1981 Phys. Rev. B 23 5048-79.

[13] Lindahl E, Hess B and van der Spoel D 2001 J. Mol. Mod. 7 306-317.

[14] Sorin E J and Pande V S 2005 Biophys. J. 88 2472-93.

[15] Jorgensen W, Chandrasekhar J and Madura J D 1983 J. Chem. Phys. 79 926-935.

[16] Parrinello M and Rahman A 1981 J. Appl. Physics 52 7182-90.

[17] Berendsen H J C, Postma J P M, DiNola A and Haak J R 1984 J. Chem. Phys. 81 3684-90.

[18] Johnson A T C, Staii C, Chen M, Khamis S, Johnson R, Klein M L and Gelperin A 2006 Semicond. Sci. Technol. 21 S17-S21.

[19] Atomistix v.2008.10, www.quantumwise.com.

[20] Roman C, Ciontu F and Courtois B 2004 Proc. of the European Micro and Nano Systems 2004 (EMN04) (Paris, France) pp 273-278.

[21] Simeoni M, Picozzi S and Delley B 2004 Surf. Sci. 562 43-52.

[22] Abadir G B, Walus K, Turner R F B and Pulfrey D L 2008 IJHSES 18 879-887.

[23] Abadir G B, Walus K and Pulfrey D L 2009 Appl. Phys. Lett. 94176101.

[24] Abadir G B, Walus K and Pulfrey D L 2009 J. Comp. Elec. 8 1-9.

[25] Abadir G B, Walus K, Turner R F B and Pulfrey D L 2008 Proc. of the 8th IEEE Conference on Nanotechnology (Arlington, Texas) pp 230-232.

[26] Star A, Han T, Gabriel J P, Bradley K, and Gruner G 2003 Nano Lett. 3 1421-23.

[27] Gokturk H 2005 Proc. of the 5th IEEE Conference on Nanotechnology (Nagoya, Japan) pp 677-680.

[28] Albrecht P M, Barraza-Lopez S, and Lyding J W 2007 Nanotech. 18 1-7.

[29] Whitford D 2005 Proteins: Structure and Function (John Wiley \& Sons: Chichester, West Sussex) p 28.

[30] Park J Y, Rosenblatt S, Yaish Y, Sazanova V, Ustunel H, Braig S, Arias T A, Brouwer P W, McEuen P L 2004, Nano. Lett. 4 517-520.

[31] Javey A, Guo J, Paulsson M, Wang Q, Mann D, Lundstrom M and Dai H 2004 Phys. Rev. Lett. 92106804.

[32] Seidel R V, Graham A P, Rajasekharan B, Unger E, Liebau M, Duesberg G S, Kreupl F, and Hoenlein W 2004 J. Appl. Phys. 96 6694-99.

[33] Collins P G, Hersam M, Arnold M, Martel R and Avouris P 2001 Phys. Rev. Lett. 86 3128-31. 